\title{
The ARAUCARIA project. Discovery of Cepheid Variables in NGC 300 from a Wide-Field Imaging Survey ${ }^{\circ}$
}

\author{
Grzegorz Pietrzyński \\ Universidad de Concepción, Departamento de Fisica, Grupo de Astronomia, Casilla 160-C, \\ Concepción, Chile \\ Warsaw University Observatory, Al. Ujazdowskie 4,00-478, Warsaw, Poland \\ Wolfgang Gieren \\ Universidad de Concepción, Departamento de Fisica, Grupo de Astronomia, Casilla 160-C, \\ Concepción, Chile \\ Pascal Fouqué \\ Observatoire de Paris-Meudon DESPA, F-92195 Meudon CEDEX, France \\ European Southern Observatory, Casilla 19001, Santiago 19, Chile \\ Frederic Pont \\ Universidad de Chile, Departamento de Astronomia, Casilla 36D, Santiago, Chile
}

\begin{abstract}
We have conducted a survey for Cepheid variables in the Sculptor Group spiral NGC 300. Based on observations obtained with the Wide-Field Camera at the $2.2 \mathrm{~m} \mathrm{ESO/MPI} \mathrm{telescope} \mathrm{during} 29$ nights spread over a 5.3 month interval, 117 Cepheids and 12 Cepheid candidates were found which cover the period range from 115 to 5.4 days. We present a catalog which provides equatorial coordinates, period, time of maximum brightness, and intensity mean B and $\mathrm{V}$ magnitudes for each variable, and we show phased $\mathrm{B}$ and $\mathrm{V}$ light curves for all the Cepheids found. We also present the individual B and V observations for each Cepheid in our catalog. During our search we rediscovered all 18 previously known Cepheids, and confirmed the Cepheid nature of 3 Cepheid candidates from the previous photographic survey of Graham. Star V4 in Graham's list, classified by him as an eclipsing binary, turns out to be another Cepheid.

We find very good agreement between our photometry and that obtained by Freedman et al. from ground-based CCD data for common stars. Using the
\end{abstract}

\footnotetext{
${ }^{1}$ Based on observations obtained with the $2.2 \mathrm{~m}$ ESO/MPI telescope at the European Southern Observatory
} 
earlier data together with our new data, we were able to significantly improve the periods for 15 Cepheids in our sample. One of the Cepheids with earlier observations shows evidence for a change of its period over the last 26 years. The Cepheids delineate the spiral arms of NGC 300, and a couple of them were detected very close to the center of the galaxy. From the color-magnitude diagram of NGC 300 constructed from our data, we expect that our Cepheid detection is near-complete for variables with periods larger than about 10 days.

We present plots of the PL relations in the $\mathrm{B}$ and $\mathrm{V}$ bands obtained from our data, which clearly demonstrate the presence of a Malmquist bias for periods below about 10 days. A thorough discussion of the distance to NGC 300 will be presented in a forthcoming paper which will include the analysis of photometry in longer-wavelength bands.

Subject headings: galaxies:individual (NGC300) - galaxies: stellar content stars: variable - stars: Cepheids - galaxies: distances and redshifts

\section{Introduction}

One of the most fascinating and challenging chapters of modern astrophysics has been the effort to establish the extragalactic distance scale with an ever increasing accuracy. Key to this effort has been the use of the Hubble Space Telescope over the last years which has allowed to detect and measure the brightness of Cepheid variables in some 20 nearby spiral galaxies and determine their distances from the Cepheid period-luminosity relation. In fact, the HST Key Project team on the extragalactic distance scale has just recently presented their final results for the Cepheid distances to their selected galaxies (Freedman et al. 2001), from which secondary methods of distance measurement have yielded an improved determination of the Hubble constant. However, in the HST Key Project procedure to determine the Hubble constant there are still a number of systematic uncertainties which prevent, at the present time, a truly accurate determination of this constant which is so important to cosmology. The most nagging problem continues to be the distance to the LMC, whose Cepheids are used as a fiducial to determine the relative distances to the other galaxies. The distance to the LMC is currently the largest single contributor to the systematic uncertainty on $\mathrm{H}_{0}$ (Mould et al. 2000). Another problem, affecting the calibrating Cepheid samples in different galaxies, is the dependence of Cepheid absolute magnitudes on metallicity. Currently there are very controversial claims about the importance of this effect for the determination of Cepheid distances to galaxies, and a 
truly accurate empirical determination of the effect is urgently needed to further reduce the uncertainty on the Hubble constant.

In spite of the very important progress achieved over the last years, not only by HST but by many other teams of researchers using ground-based telescopes, it seems clear that the remaining uncertainty in the distance scale can only be significantly reduced if we succeed to make true progress in determining the distance to the LMC, and a number of other nearby galaxies. To achieve this, we must learn more about the systematic uncertainties in the principal methods currently used to find these distances. The most promising stellar methods, apart from Cepheid variables, use red clump giants, the tip of the red giant branch, eclipsing binaries, and planetary nebulae as distance indicators. Another, relatively new tool to determine extragalactic distances from individual stars is the wind momentum-luminosity relationship (WLR) for blue supergiants (Kudritzki et al. 1999; Bresolin et al. 2001). Only when the true capabilities and systematics of these various methods of distance determination are fully understood, the techniques properly calibrated, and agreement on the distances to a number of nearby galaxies with different environmental properties from all these methods is achieved, can we trust that the local calibration of the distance scale has been finally settled. A working group at the Universidad de Concepción (including several of the authors of this paper) has recently been established to work on the improvement of stellar distance indicators. We call our project the ARAUCARIA project (making reference to a famous tree at our southern location), and we hope to make a significant contribution to the improved calibration of the local extragalactic distance scale, over the next years.

Our target to start with is the Sculptor Group spiral galaxy NGC 300. At a distance of about 2.0 Mpc (Freedman et al. 2001), this galaxy is sufficiently close to resolve its stellar populations. Since NGC 300 shows clear signs of recent, massive star formation, it seemed an excellent target to discover a significant number of Cepheid variables. Indeed, a relatively small number of Cepheids in NGC 300 had already been previously discovered by Graham (1984) with photographic techniques at the CTIO $4 \mathrm{~m}$ telescope, and a subsample of these variables was later observed with a ground-based CCD camera by Freedman et al. (1992). However, taking advantage of new wide-field imaging capabilities, we hoped to find many more Cepheids and determine their properties with a higher accuracy than what had been achieved in former work, a hope which is borne out by the results presented in this paper. In a parallel project, we are conducting a survey for blue supergiant stars in the disc of NGC 300 with the ESO VLT telescope; first results have recently been presented in Bresolin et al. (2001). The blue supergiants will be used to establish the (stellar) metallicity gradient in the disc of NGC 300 to perform a new and accurate empirical calibration of the effect of metallicity on Cepheid luminosities, one of the principal goals of our current 
project. They will also be used to improve the WLR calibration for blue supergiants. In addition, we have also secured narrow-band images of the galaxy to conduct a complete survey for its planetary nebulae population and improve the calibration of the PNLF method of distance determination.

The present paper is organized as follows. In section 2, we describe the observations from which the current survey for Cepheids was conducted. In section 3 we describe the reduction techniques and calibration of the images. In section 4, we present the catalog of newly discovered Cepheid variables in NGC 300 (variable stars of different types will be presented in a forthcoming paper). In section 5, we compare our new data to the ones for those Cepheids for which previous photometry exists from the work of Graham, and of Freedman et al. (1992). A discussion of the results and conclusions are presented in section 6.

\section{Observations}

All observations presented in this paper have been collected with the ESO/MPG $2.2 \mathrm{~m}$ telescope at the La Silla observatory in Chile. The telescope was equipped with the ESO mosaic Wide Field Camera (WFI) consisting of eight 2048 x 4098 pixel arrays. The CCDs were separated by gaps of 23.8 and 14.3 arcsec in right ascension and declination directions, respectively. The total field of view was about 34 x 33 arcmin with a scale of 0.238 arcsec / pixel.

Our observations started on July 31, 1999 and lasted until January 8, 2001. During this period, we secured $150 \mathrm{~B}, 138 \mathrm{~V}, 49 \mathrm{R}$ and 145 I-band images centered on NGC 300, on 29 different nights allocated to our programme. Most of these nights were photometric, and the seeing ranged from 0.7 to 1.5 arcsec during the vast majority of the exposures taken. In order to obtain photometry for all stars from the whole observed field including those located in the gaps between CCDs, the observations were carried out in a "dithering" mode. During a given night 5 consecutive 360 s exposures, slightly shifted in right ascension and/or declination with respect to each other, were made in a given filter. This technique also allowed us to obtain a better accuracy of the photometric measurements by evaluating a mean from all observations taken during a given night through a given filter, and help to recognize bad measurements caused by weather, blending, or presence of bad pixels. On about half of the nights we obtained full observing sequences through BVRI filters, in the other nights we could only observe BVR or BVI sequences. On a few nights, only BV sequences could be obtained. Effectively, 23-30 epochs, depending on the brightness of a given star and filter, were obtained. The journal of B and V observations which were used 
to conduct the search for Cepheids is presented in Table 1.

\section{Reductions and Calibrations}

Preliminary reductions (i.e. debiasing and flatfielding) were carried out in the standard way with the IRAF' package. In order to avoid possible variations of the PSF across the image each of the 8 chips was divided into 2 slightly overlapping subframes. Then profile photometry for all stars detected on all subframes was performed with the DAOPHOT and ALLSTAR programs. The PSF model was derived iteratively. First, 10 relatively bright and isolated stars were selected and the first approximation of the PSF model was calculated. In the next step we subtracted all neighbor stars with the ALLSTAR program and derived the PSF model again. The PSF model obtained after three such loops was finally adopted. The photometric data from each two subframes were finally tied together using stars located in the overlapping regions.

In order to prepare an unique system of identification of stars in the observed field, the best (i.e. obtained under the best seeing conditions) set of five $\mathrm{V}$ images was selected and stacked together. The resulting "template" included all stars located in the gaps between chips and covered a region of about 34 x 33 arcmin (see Fig. 1). The reference list with coordinates of objects from the template image was prepared with the DAOPHOT program. After rejecting evidently spurious detections due to saturated stars or wild pixels, the total number of objects we detected was about 32000. The pixel coordinates of all stars from a given field were transformed to the template coordinate system, and a unique number corresponding to the numbering system from the reference list was assigned to each object.

A detailed description of the adopted photometric calibration procedure, completeness tests, a comparison with previous results together with a presentation and discussion of CMDs will be presented in a forthcoming paper. Briefly, to calibrate the photometric magnitudes of all stars on the wide field images an extensive sequence of secondary standard stars, distributed over almost the whole observed area and spanning a broad range in magnitudes and colors, was set up (Pietrzyński et al. 2001). The WFI photometry was transformed to the standard system using about 100 stars from this list. Derived residuals usually did not exceed $0.04 \mathrm{mag}$ and did not show any dependence on color or brightness. The accuracy of the zero point of our WFI photometry is about $0.03 \mathrm{mag}$

\footnotetext{
${ }^{2}$ IRAF is distributed by the National Optical Astronomy Observatories, which are operated by the Association of Universities for Research in Astronomy, Inc., under cooperative agreement with the NSF.
} 
Table 1. Journal of Observations

\begin{tabular}{|c|c|c|}
\hline UT date & Filter & HJD \\
\hline 1999 July 31 & B & 2451390.780 \\
\hline 1999 August 1 & B & 2451391.936 \\
\hline 1999 August 1 & V & 2451391.846 \\
\hline 1999 August 2 & B & 2451392.766 \\
\hline 1999 August 3 & B & 2451393.819 \\
\hline 1999 August 3 & $\mathrm{~V}$ & 2451393.847 \\
\hline 1999 August 10 & B & 2451400.907 \\
\hline 1999 August 10 & $\mathrm{~V}$ & 2451400.806 \\
\hline 1999 August 14 & B & 2451404.807 \\
\hline 1999 August 14 & $\mathrm{~V}$ & 2451404.867 \\
\hline 1999 August 18 & B & 2451408.812 \\
\hline 1999 August 18 & $\mathrm{~V}$ & 2451408.840 \\
\hline 1999 August 31 & B & 2451421.882 \\
\hline 1999 August 31 & $\mathrm{~V}$ & 2451421.861 \\
\hline 1999 September 3 & B & 2451424.811 \\
\hline 1999 September 3 & $\mathrm{~V}$ & 2451424.839 \\
\hline 1999 September 10 & B & 2451431.797 \\
\hline 1999 September 10 & V & 2451431.824 \\
\hline 1999 September 12 & B & 2451433.793 \\
\hline 1999 September 12 & $\mathrm{~V}$ & 2451433.822 \\
\hline 1999 September 15 & B & 2451436.799 \\
\hline 1999 September 15 & $\mathrm{~V}$ & 2451436.828 \\
\hline 1999 September 17 & B & 2451438.804 \\
\hline 1999 September 17 & $\mathrm{~V}$ & 2451438.832 \\
\hline 1999 October 30 & B & 2451481.576 \\
\hline 1999 October 30 & $\mathrm{~V}$ & 2451481.603 \\
\hline 1999 November 2 & B & 2451484.575 \\
\hline 1999 November 2 & $\mathrm{~V}$ & 2451484.632 \\
\hline 1999 November 4 & V & 2451486.577 \\
\hline 1999 November 6 & B & 2451488.535 \\
\hline 1999 November 6 & $\mathrm{~V}$ & 2451488.563 \\
\hline 1999 November 9 & B & 2451491.555 \\
\hline 1999 November 9 & $\mathrm{~V}$ & 2451491.580 \\
\hline
\end{tabular}


Table 1. Journal of Observations - Concluded

\begin{tabular}{|c|c|c|}
\hline UT date & Filter & HJD \\
\hline 1999 November 14 & B & 2451496.506 \\
\hline 1999 November 14 & $\mathrm{~V}$ & 2451496.560 \\
\hline 1999 November 16 & B & 2451498.554 \\
\hline 1999 November 16 & $\mathrm{~V}$ & 2451498.582 \\
\hline 1999 December 4 & B & 2451516.584 \\
\hline 1999 December 4 & $\mathrm{~V}$ & 2451516.611 \\
\hline 1999 December 7 & B & 2451519.560 \\
\hline 1999 December 7 & V & 2451519.585 \\
\hline 1999 December 9 & B & 2451521.561 \\
\hline 1999 December 9 & $\mathrm{~V}$ & 2451521.587 \\
\hline 1999 December 12 & B & 2451524.564 \\
\hline 1999 December 12 & $\mathrm{~V}$ & 2451524.591 \\
\hline 1999 December 14 & B & 2451526.539 \\
\hline 1999 December 14 & $\mathrm{~V}$ & 2451526.564 \\
\hline 1999 December 16 & B & 2451528.593 \\
\hline 1999 December 16 & $\mathrm{~V}$ & 2451528.566 \\
\hline 1999 December 19 & B & 2451531.561 \\
\hline 1999 December 19 & $\mathrm{~V}$ & 2451531.590 \\
\hline 1999 December 31 & B & 2451543.536 \\
\hline 1999 December 31 & V & 2451543.562 \\
\hline 2000 January 2 & B & 2451545.624 \\
\hline 2000 January 4 & B & 2451547.549 \\
\hline 2000 January 4 & $\mathrm{~V}$ & 2451547.601 \\
\hline 2000 January 9 & B & 2451552.534 \\
\hline 2000 January 9 & $\mathrm{~V}$ & 2451552.564 \\
\hline
\end{tabular}


in the standard $\mathrm{V}$ and $\mathrm{B}$ bands. We found good agreement (the mean differences were $\Delta V=0.008 \pm 0.028 \mathrm{mag}, \Delta(B-V)=-0.018 \pm 0.031 \mathrm{mag})$ between our photometry and that obtained by Walker (1995), for 22 common stars in both samples.

Taking into account the periods of the potential Cepheids, all consecutive observations obtained in a given filter, during a given night (amounting to typically 30 minutes) still define practically the same phase in the light curve, even for the shortest-period Cepheids we detected (see section 4). For that reason we decided to improve the accuracy of our Cepheid measurements, for a given night and in a given filter, by adopting the mean magnitude from the individual dithered observations.

Equatorial coordinates were calculated with the algorithm developed and used by the OGLE team (Udalski et al. 1998). Shortly, a FITS file slightly larger than our template was extracted from the Digital Sky Survey (DSS) images and all stars having more than 200 counts above the sky level were detected on it. The pixel $(\mathrm{x}, \mathrm{y})$ coordinates of the detected stars were transformed to equatorial coordinates, and then to $\left(\mathrm{x}^{\prime}, \mathrm{y}^{\prime}\right)$ pixel coordinates on the plane tangential to the celestial sphere at the center of our field. The resulting two sets of pixel coordinates were tied together using third order polynomials. The internal accuracy of our transformation was about 0.3 arcsec.

\section{The Cepheid Catalog}

As a first step, all stars were subjected to a period search using the AoV algorithm (Schwarzenberg-Czerny 1989). We searched for periods between 0.2 and 90 days, the latter value corresponding to about a half of the time span of our observations. For the detected Cepheids, the accuracy of the derived periods is about $0.5-1 \times 10^{-2} \times \mathrm{P}$, or roughly 1 percent of the period lengths. However, for some of the Cepheids, the ones with previous observations from Freedman et al. (1992) and/or Graham (1984), we were able to determine

much more accurate periods by combining the different sets of photometric measurements (see chapter 5 for more details).

In order to distinguish Cepheid variables from other types of variable stars we used the following selection criteria:

1. Stars must exhibit the typical asymmetrical Cepheid-like shape of the light curves

2. $\mathrm{A}_{\mathrm{B}}>0.4 \mathrm{mag}$

3. $A_{B}>A_{V}$

4. $0.4<\mathrm{B}-\mathrm{V}<1.5$ 
Here, $A_{B}$ and $A_{V}$ stand for the amplitudes in the $B$ and $V$ bands, respectively. Regarding the color selection criterion, we note that the foreground reddening in the direction to NGC 300 is only $E(B-V)=0.02$ (Burstein \& Heiles 1984) so that the observed colors correspond closely to the intrinsic colors of the stars, supposing that intrinsic absorption within NGC 300 is small.

To derive the mean magnitudes for those variables passing the selection test for a Cepheid, the light curves were approximated by Fourier series of an order ranging from 2 to 5 , depending on the accuracy of the photometry and/or the phase coverage. Fig. 2 shows such fits for two exemplary B light curves: one of a bright, well observed Cepheid, and one of a much fainter Cepheid having a noisier light curve and less points because the star could only be detected and measured on nights of good seeing. However, even in such cases mean magnitudes could be determined rather accurately. They were obtained by fitting the light curves, converted to intensity units, and transforming the mean intensities back to a magnitude scale. The statistical accuracy of our intensity mean B and $\mathrm{V}$ magnitudes was typically better than $0.01 \mathrm{mag}$ (i.e. much smaller than the uncertainty of the zero point of our photometry).

Alltogether, 117 stars satisfied our selection criteria and entered our catalog of Cepheids presented in this paper. Table 2 contains their description. The first column is the star identification number. In the next columns the equatorial coordinates, derived periods, epochs of zero phase corresponding to maximum light in $\mathrm{V}$, and intensity mean brightnesses in the $\mathrm{B}$ and $\mathrm{V}$ bands are given. The last column contains remarks on the objects. V2, V3, etc correspond to the numbering system introduced by Graham (1984) for the 34 variable star candidates in NGC 300 discovered by him. The digits of the periods given reflect their uncertainties. The phased B and V light curves for all Cepheids are presented in Fig. 3. Note that each observational point on the light curves is the mean from all observations (up to 5) obtained through a given filter during a particular night.

In addition to the 117 Cepheids in Table 2, we present another 12 Cepheid candidates in Table 3. These objects do not satisfy all criteria described above, but the shapes of their (noisy) light curves suggest that they may be Cepheids, too. The phased B and V light curves of these objects are displayed in Fig. 4. For all Cepheids, the individual B and V observations are given in Table 4.

Finding charts for all Cepheid variables, prepared based on $\mathrm{V}$ band image are presented in Figure 5 (available only in the electronic edition of the Journal). They can be also be obtained from the authors upon request. 
Table 2. Cepheids in NGC 300

\begin{tabular}{|c|c|c|c|c|c|c|c|}
\hline ID & RA (J2000) & DEC (J2000) & $\begin{array}{c}\mathrm{P} \\
{[\text { days }]}\end{array}$ & $\begin{array}{c}\mathrm{T}_{0}-2450000 \\
{[\text { HJD] }}\end{array}$ & $\begin{array}{r}<B> \\
{[\mathrm{mag}]}\end{array}$ & $\begin{array}{r}<V> \\
{[\mathrm{mag}]}\end{array}$ & Remarks \\
\hline сер001 & $0^{\mathrm{h}} 55^{\mathrm{m}} 11.60$ & $-37^{\circ} 33^{\prime} 54^{\prime \prime} 9$ & 115 & 1487 & 21.14 & 20.08 & V24, blend \\
\hline сер002 & $0^{\mathrm{h}} 54^{\mathrm{m}} 35^{\mathrm{s}} .04$ & $-37^{\circ} 35^{\prime} 00^{\prime \prime} 6$ & 89.05 & 1503.26 & 20.78 & 19.77 & V12, blend \\
\hline cep003 & $0^{\mathrm{h}} 54^{\mathrm{m}} 54^{\mathrm{s}} \cdot 32$ & $-37^{\circ} 37^{\prime} 01^{\prime \prime} 6$ & 83 & 1404 & 20.13 & 19.26 & \\
\hline сер004 & $0^{\mathrm{h}} 54^{\mathrm{m}} 53^{\mathrm{s}} .98$ & $-37^{\circ} 39^{\prime} 30^{\prime \prime} 5$ & 75 & 1394 & 20.69 & 19.78 & V18, blend \\
\hline cep005 & $0^{\mathrm{h}} 54^{\mathrm{m}} 08^{\mathrm{s}} .90$ & $-37^{\circ} 41^{\prime} 40^{\prime \prime} 8$ & 56.57 & 1411.68 & 21.12 & 20.39 & V3 \\
\hline cep006 & $0^{\mathrm{h}} 55^{\mathrm{m}} 41.25$ & $-37^{\circ} 49^{\prime} 06^{\prime \prime} 5$ & 52.754 & 1397.017 & 21.31 & 20.47 & V32 \\
\hline cep007 & $0^{\mathrm{h}} 54^{\mathrm{m}} 23.79$ & $-37^{\circ} 41^{\prime} 04^{\prime \prime} 5$ & 43.35 & 1425.47 & 21.86 & 20.87 & V8 \\
\hline cep008 & $0^{\mathrm{h}} 54^{\mathrm{m}} 26.47$ & $-37^{\circ} 42^{\prime} 04^{\prime \prime} 8$ & 40.6 & 1545.8 & 20.96 & 20.33 & \\
\hline сер009 & $0^{\mathrm{h}} 54^{\mathrm{m}} 41.97$ & $-37^{\circ} 34^{\prime} 44^{\prime \prime} 3$ & 36.7 & 1520.1 & 21.97 & 21.06 & \\
\hline cep010 & $0^{\mathrm{h}} 54^{\mathrm{m}} 48.50$ & $-37^{\circ} 44^{\prime} 58^{\prime \prime} 4$ & 35.8 & 1531.3 & 22.44 & 21.28 & \\
\hline сер011 & $0^{\mathrm{h}} 54^{\mathrm{m}} 57^{\mathrm{s}} \cdot 23$ & $-37^{\circ} 40^{\prime} 26^{\prime \prime} 7$ & 35.3 & 1487.8 & 21.98 & 21.17 & \\
\hline сер012 & $0^{\mathrm{h}} 55^{\mathrm{m}} 16^{\mathrm{s}} 16$ & $-37^{\circ} 37^{\prime} 26^{\prime \prime} 9$ & 35.005 & 1436.958 & 21.61 & 20.86 & V27 \\
\hline сер013 & $0^{\mathrm{h}} 55^{\mathrm{m}} 12.28$ & $-37^{\circ} 42^{\prime} 44^{\prime \prime} 0$ & 34.72 & 1520.26 & 21.73 & 20.86 & V25 \\
\hline сер014 & $0^{\mathrm{h}} 54^{\mathrm{m}} 41^{\mathrm{s}} .73$ & $-37^{\circ} 46^{\prime} 15^{\prime \prime} 9$ & 33.975 & 1405.393 & 21.43 & 20.69 & V13 \\
\hline сер015 & $0^{\mathrm{h}} 54^{\mathrm{m}} 51^{\mathrm{s}} .36$ & $-37^{\circ} 39^{\prime} 11^{\prime \prime} 0$ & 32.3 & 1543.8 & 21.80 & 20.98 & \\
\hline сер016 & $0^{\mathrm{h}} 55^{\mathrm{m}} 04^{\mathrm{s}} .71$ & $-37^{\circ} 43^{\prime} 59^{\prime \prime} 0$ & 28.6 & 1529.13 & 22.33 & 21.34 & \\
\hline сер017 & $0^{\mathrm{h}} 54^{\mathrm{m}} 52^{\mathrm{s}} .64$ & $-37^{\circ} 35^{\prime} 39^{\prime \prime} 7$ & 28.5 & 1436.8 & 22.37 & 21.55 & \\
\hline cep018 & $0^{\mathrm{h}} 54^{\mathrm{m}} 26.85$ & $-37^{\circ} 34^{\prime} 23^{\prime \prime} .9$ & 25.010 & 1543.715 & 22.12 & 21.43 & V10 \\
\hline сер019 & $0^{\mathrm{h}} 55^{\mathrm{m}} 25.94$ & $-37^{\circ} 39^{\prime} 42^{\prime \prime} .5$ & 24.9 & 1528.6 & 22.48 & 21.60 & V28 \\
\hline cep020 & $0^{\mathrm{h}} 54^{\mathrm{m}} 53.78$ & $-37^{\circ} 41^{\prime} 19^{\prime \prime} 2$ & 24.4 & 1392.1 & 22.65 & 21.83 & \\
\hline cep021 & $0^{\mathrm{h}} 54^{\mathrm{m}} 59.56$ & $-37^{\circ} 41^{\prime} 29^{\prime \prime} 9$ & 24.228 & 1404.237 & 21.83 & 21.25 & V33 \\
\hline сер022 & $0^{\mathrm{h}} 55^{\mathrm{m}} 36.56$ & $-37^{\circ} 40^{\prime} 09^{\prime \prime} 0$ & 24.2 & 1496.7 & 22.36 & 21.64 & V31 \\
\hline cep023 & $0^{\mathrm{h}} 55^{\mathrm{m}} 02.25$ & $-37^{\circ} 45^{\prime} 49^{\prime \prime} 2$ & 24.033 & 1484.147 & 22.83 & 21.87 & V21 \\
\hline сер024 & $0^{\mathrm{h}} 54^{\mathrm{m}} 45^{\mathrm{s}} .52$ & $-37^{\circ} 42^{\prime} 18^{\prime \prime} .5$ & 23.8 & 1496.4 & 21.87 & 21.12 & \\
\hline cep025 & $0^{\mathrm{h}} 54^{\mathrm{m}} 25.97$ & $-37^{\circ} 40^{\prime} 38^{\prime \prime} .7$ & 23.7 & 1391.7 & 22.49 & 21.64 & \\
\hline сер026 & $0^{\mathrm{h}} 55^{\mathrm{m}} 33.14$ & $-37^{\circ} 36^{\prime} 17^{\prime \prime} 1$ & 23.446 & 1408.859 & 21.85 & 21.21 & V29 \\
\hline cep027 & $0^{\mathrm{h}} 55^{\mathrm{m}} 12.10$ & $-37^{\circ} 37^{\prime} 42^{\prime \prime} .5$ & 23.3 & 1491.6 & 22.03 & 21.25 & \\
\hline cep028 & $0^{\mathrm{h}} 54^{\mathrm{m}} 51^{\mathrm{s}} 39$ & $-37^{\circ} 39^{\prime} 27^{\prime \prime} 0$ & 23.1 & 1487.8 & 21.59 & 21.02 & \\
\hline cep029 & $0^{\mathrm{h}} 54^{\mathrm{m}} 04^{\mathrm{s}} .52$ & $-37^{\circ} 39^{\prime} 20^{\prime \prime} 5$ & 22.6 & 1392. & 22.64 & 21.83 & \\
\hline cep030 & $0^{\mathrm{h}} 54^{\mathrm{m}} 46.63$ & $-37^{\circ} 37^{\prime} 14^{\prime \prime} 2$ & 22.3 & 1517.5 & 22.35 & 21.53 & \\
\hline cep031 & $0^{\mathrm{h}} 55^{\mathrm{m}} 32^{\mathrm{s}} .91$ & $-37^{\circ} 40^{\prime} 45^{\prime \prime} 0$ & 21.5 & 1407.5 & 23.03 & 21.95 & \\
\hline cep032 & $0^{\mathrm{h}} 54^{\mathrm{m}} 59.48$ & $-37^{\circ} 38^{\prime} 34^{\prime \prime} 2$ & 21.1 & 1496.6 & 22.09 & 21.41 & \\
\hline сер033 & $0^{\mathrm{h}} 55^{\mathrm{m}} 07^{\mathrm{s}} .02$ & $-37^{\circ} 39^{\prime} 40^{\prime \prime} .5$ & 20.7 & 1401.0 & 22.32 & 21.74 & \\
\hline сер034 & $0^{\mathrm{h}} 55^{\mathrm{m}} 02^{\mathrm{s}} .54$ & $-37^{\circ} 33^{\prime} 18^{\prime \prime} 8$ & 20.64 & 1390.76 & 22.50 & 21.78 & V22 \\
\hline
\end{tabular}


Table 2-Continued

\begin{tabular}{|c|c|c|c|c|c|c|c|}
\hline ID & RA (J2000) & DEC (J2000) & $\begin{array}{c}\mathrm{P} \\
{[\text { days }]}\end{array}$ & $\begin{array}{c}\mathrm{T}_{0}-2450000 \\
\text { [HJD] }\end{array}$ & $\begin{array}{r}<B> \\
{[\mathrm{mag}]}\end{array}$ & $\begin{array}{l}<V> \\
{[\mathrm{mag}]}\end{array}$ & Remarks \\
\hline сер035 & $0^{\mathrm{h}} 54^{\mathrm{m}} 44^{\mathrm{s}} .65$ & $-37^{\circ} 38^{\prime} 30^{\prime \prime} 6$ & 19.4 & 1519.3 & 22.35 & 21.46 & \\
\hline cep036 & $0^{\mathrm{h}} 55^{\mathrm{m}} 08^{\mathrm{s}} \cdot 33$ & $-37^{\circ} 38^{\prime} 11^{\prime \prime} 8$ & 18.9 & 1488.6 & 23.25 & 22.47 & \\
\hline cep037 & $0^{\mathrm{h}} 54^{\mathrm{m}} 37^{\mathrm{s}} .07$ & $-37^{\circ} 39^{\prime} 05^{\prime \prime} .5$ & 18.7 & 1545.6 & 23.37 & 22.43 & \\
\hline cep038 & $0^{\mathrm{h}} 55^{\mathrm{m}} 31^{\mathrm{s}} \cdot 34$ & $-37^{\circ} 34^{\prime} 31^{\prime \prime} 4$ & 18.2 & 1408.9 & 22.12 & 21.53 & \\
\hline сер039 & $0^{\mathrm{h}} 55^{\mathrm{m}} 04^{\mathrm{s}} .12$ & $-37^{\circ} 46^{\prime} 14^{\prime \prime} 1$ & 18.2 & 1391.4 & 23.05 & 22.13 & \\
\hline cep040 & $0^{\mathrm{h}} 54^{\mathrm{m}} 25.00$ & $-37^{\circ} 37^{\prime} 57^{\prime \prime} .7$ & 18.213 & 1545.589 & 22.00 & 21.49 & V9 \\
\hline cep041 & $0^{\mathrm{h}} 55^{\mathrm{m}} 01^{\mathrm{s}} .94$ & $-37^{\circ} 45^{\prime} 45^{\prime \prime} 0$ & 18.012 & 1392.004 & 22.00 & 21.43 & V20 \\
\hline cep042 & $0^{\mathrm{h}} 53^{\mathrm{m}} 37^{\mathrm{s}} \cdot 17$ & $-37^{\circ} 26^{\prime} 00^{\prime \prime} 6$ & 17.93 & 1408.87 & 22.88 & 22.02 & \\
\hline cep043 & $0^{\mathrm{h}} 54^{\mathrm{m}} 02^{\mathrm{s}} \cdot 20$ & $-37^{\circ} 39^{\prime} 02^{\prime \prime} .2$ & 17.833 & 1524.816 & 22.07 & 21.51 & V2 \\
\hline сер044 & $0^{\mathrm{h}} 54^{\mathrm{m}} 59.73$ & $-37^{\circ} 43^{\prime} 03^{\prime \prime} 0$ & 17.25 & 1408.30 & 22.89 & 22.06 & \\
\hline cep045 & $0^{\mathrm{h}} 55^{\mathrm{m}} 29.33$ & $-37^{\circ} 41^{\prime} 23^{\prime \prime} 0$ & 17.0 & 1548.0 & 22.16 & 21.66 & \\
\hline сер046 & $0^{\mathrm{h}} 55^{\mathrm{m}} 21^{\mathrm{s}} 40$ & $-37^{\circ} 33^{\prime} 48^{\prime \prime} 3$ & 16.5 & 1392.9 & 23.34 & 22.47 & \\
\hline cep047 & $0^{\mathrm{h}} 54^{\mathrm{m}} 39.67$ & $-37^{\circ} 42^{\prime} 38^{\prime \prime} .7$ & 16.5 & 1496.6 & 23.44 & 22.21 & \\
\hline cep048 & $0^{\mathrm{h}} 55^{\mathrm{m}} 35^{\mathrm{s}} .68$ & $-37^{\circ} 44^{\prime} 12^{\prime \prime} 1$ & 16.5 & 1390.6 & 22.12 & 21.51 & \\
\hline сер049 & $0^{\mathrm{h}} 55^{\mathrm{m}} 14^{\mathrm{s}} .64$ & $-37^{\circ} 43^{\prime} 37^{\prime \prime} 6$ & 16.2 & 1390.8 & 23.00 & 22.07 & \\
\hline cep050 & $0^{\mathrm{h}} 54^{\mathrm{m}} 30.22$ & $-37^{\circ} 43^{\prime} 04^{\prime \prime} 4$ & 15.9 & 1438.2 & 22.39 & 21.74 & \\
\hline cep051 & $0^{\mathrm{h}} 54^{\mathrm{m}} 22^{\mathrm{s}} .63$ & $-37^{\circ} 34^{\prime} 48^{\prime \prime} 0$ & 15.72 & 1543.42 & 22.64 & 22.00 & \\
\hline сер052 & $0^{\mathrm{h}} 55^{\mathrm{m}} 22^{\mathrm{s}} .01$ & $-37^{\circ} 34^{\prime} 22^{\prime \prime} 6$ & 15.6 & 1498.8 & 22.22 & 21.55 & V26 \\
\hline cep053 & $0^{\mathrm{h}} 55^{\mathrm{m}} 20.84$ & $-37^{\circ} 43^{\prime} 03^{\prime \prime} .0$ & 15.5 & 1520.5 & 23.11 & 22.21 & \\
\hline cep054 & $0^{\mathrm{h}} 54^{\mathrm{m}} 09.33$ & $-37^{\circ} 40^{\prime} 51^{\prime \prime} 8$ & 15.4 & 1481.4 & 22.21 & 21.70 & V4 \\
\hline cep055 & $0^{\mathrm{h}} 55^{\mathrm{m}} 06.92$ & $-37^{\circ} 44^{\prime} 36^{\prime \prime} 4$ & 15.0 & 1404.9 & 22.24 & 21.66 & \\
\hline сер056 & $0^{\mathrm{h}} 54^{\mathrm{m}} 37^{\mathrm{s}} \cdot 36$ & $-37^{\circ} 38^{\prime} 05^{\prime \prime} 8$ & 15.05 & 1431.95 & 22.77 & 22.22 & \\
\hline сер057 & $0^{\mathrm{h}} 55^{\mathrm{m}} 08^{\mathrm{s}} 19$ & $-37^{\circ} 35^{\prime} 09^{\prime \prime} 6$ & 14.8 & 1485.3 & 22.91 & 22.17 & \\
\hline сер058 & $0^{\mathrm{h}} 55^{\mathrm{m}} 02.26$ & $-37^{\circ} 47^{\prime} 00^{\prime \prime} 6$ & 14.80 & 1519.48 & 23.00 & 22.14 & \\
\hline cep059 & $0^{\mathrm{h}} 54^{\mathrm{m}} 48^{\mathrm{s}} .55$ & $-37^{\circ} 36^{\prime} 43^{\prime \prime} 0$ & 14.5 & 1392.7 & 22.97 & 22.10 & \\
\hline cep060 & $0^{\mathrm{h}} 55^{\mathrm{m}} 06.98$ & $-37^{\circ} 41^{\prime} 32^{\prime \prime} .9$ & 14.40 & 1425.23 & 23.16 & 22.20 & \\
\hline cep061 & $0^{\mathrm{h}} 55^{\mathrm{m}} 18^{\mathrm{s}} .96$ & $-37^{\circ} 39^{\prime} 11^{\prime \prime} 3$ & 14.3 & 1519.4 & 22.99 & 22.24 & \\
\hline cep062 & $0^{\mathrm{h}} 55^{\mathrm{m}} 06^{\mathrm{s}} .82$ & $-37^{\circ} 35^{\prime} 13^{\prime \prime} 4$ & 14.3 & 1436.6 & 22.76 & 22.09 & \\
\hline cep063 & $0^{\mathrm{h}} 54^{\mathrm{m}} 12^{\mathrm{s}} \cdot 35$ & $-37^{\circ} 39^{\prime} 19^{\prime \prime} 1$ & 14.3 & 1392.8 & 22.64 & 21.98 & V7 \\
\hline cep064 & $0^{\mathrm{h}} 54^{\mathrm{m}} 53.97$ & $-37^{\circ} 44^{\prime} 42^{\prime \prime} .5$ & 14.3 & 1392.5 & 23.62 & 22.33 & \\
\hline cep065 & $0^{\mathrm{h}} 55^{\mathrm{m}} 20.28$ & $-37^{\circ} 39^{\prime} 38^{\prime \prime} .6$ & 14.1 & 1424.9 & 22.45 & 21.81 & \\
\hline cep066 & $0^{\mathrm{h}} 55^{\mathrm{m}} 29^{\mathrm{s}} .53$ & $-37^{\circ} 42^{\prime} 08^{\prime \prime} .4$ & 13.8 & 1392.7 & 22.77 & 22.10 & \\
\hline cep067 & $0^{\mathrm{h}} 54^{\mathrm{m}} 20^{\mathrm{s}} 01$ & $-37^{\circ} 38^{\prime} 32^{\prime \prime} 8$ & 13.8 & 1491.5 & 23.32 & 22.45 & \\
\hline сер068 & $0^{\mathrm{h}} 54^{\mathrm{m}} 29^{\mathrm{s}} 75$ & $-37^{\circ} 40^{\prime} 46^{\prime \prime} 1$ & 13.8 & 1437.5 & 23.10 & 22.49 & \\
\hline
\end{tabular}


Table 2-Continued

\begin{tabular}{|c|c|c|c|c|c|c|c|}
\hline ID & RA (J2000) & DEC (J2000) & $\begin{array}{c}\mathrm{P} \\
{[\text { days] }}\end{array}$ & $\begin{array}{c}\mathrm{T}_{0}-2450000 \\
{[\text { HJD }]}\end{array}$ & $\begin{array}{r}<B> \\
{[\mathrm{mag}]}\end{array}$ & $\begin{array}{r}<V> \\
{[\mathrm{mag}]}\end{array}$ & Remarks \\
\hline сер069 & $0^{\mathrm{h}} 54^{\mathrm{m}} 48.86$ & $-37^{\circ} 37^{\prime} 21^{\prime \prime} 1$ & 13.6 & 1496.6 & 22.93 & 22.30 & \\
\hline сер070 & $0^{\mathrm{h}} 54^{\mathrm{m}} 41^{\mathrm{s}} \cdot 40$ & $-37^{\circ} 33^{\prime} 48^{\prime \prime} 1$ & 13.57 & 1408.64 & 22.57 & 22.05 & \\
\hline cep071 & $0^{\mathrm{h}} 54^{\mathrm{m}} 37^{\mathrm{s}} \cdot 35$ & $-37^{\circ} 37^{\prime} 32^{\prime \prime} 4$ & 13.54 & 1438.83 & 22.49 & 21.87 & \\
\hline cep072 & $0^{\mathrm{h}} 54^{\mathrm{m}} 49.87$ & $-37^{\circ} 36^{\prime} 57^{\prime \prime} \cdot 7$ & 13.5 & 1547.9 & 23.23 & 22.33 & \\
\hline cep073 & $0^{\mathrm{h}} 54^{\mathrm{m}} 34^{\mathrm{s}} .77$ & $-37^{\circ} 39^{\prime} 53^{\prime \prime} .9$ & 13.4 & 1543.7 & 22.59 & 21.97 & \\
\hline cep074 & $0^{\mathrm{h}} 54^{\mathrm{m}} 23^{\mathrm{s}} .07$ & $-37^{\circ} 35^{\prime} 42^{\prime \prime} .2$ & 13.3 & 1438.7 & 22.52 & 21.89 & \\
\hline cep075 & $0^{\mathrm{h}} 54^{\mathrm{m}} 14^{\mathrm{s}} .77$ & $-37^{\circ} 25^{\prime} 48^{\prime \prime} .2$ & 13.3 & 1526.1 & 22.77 & 22.13 & blend \\
\hline сер076 & $0^{\mathrm{h}} 54^{\mathrm{m}} 19.58$ & $-37^{\circ} 30^{\prime} 24^{\prime \prime} 4$ & 13.1 & 1519.6 & 22.53 & 21.86 & \\
\hline cep077 & $0^{\mathrm{h}} 55^{\mathrm{m}} 15.09$ & $-37^{\circ} 34^{\prime} 33^{\prime \prime} 4$ & 13.0 & 1484.5 & 22.78 & 22.21 & \\
\hline сер078 & $0^{\mathrm{h}} 54^{\mathrm{m}} 26.76$ & $-37^{\circ} 44^{\prime} 12^{\prime \prime} .5$ & 12.5 & 1436.8 & 23.04 & 22.47 & \\
\hline сер079 & $0^{\mathrm{h}} 55^{\mathrm{m}} 07^{\mathrm{s}} \cdot 32$ & $-37^{\circ} 40^{\prime} 01^{\prime \prime} 8$ & 11.9 & 1518.5 & 22.56 & 22.06 & \\
\hline сер080 & $0^{\mathrm{h}} 54^{\mathrm{m}} 59^{\mathrm{s}}: 33$ & $-37^{\circ} 44^{\prime} 25^{\prime \prime} 6$ & 11.8 & 1431.8 & 22.34 & 21.70 & \\
\hline сер081 & $0^{\mathrm{h}} 54^{\mathrm{m}} 19.32$ & $-37^{\circ} 44^{\prime} 54^{\prime \prime} 1$ & 11.6 & 1481.6 & 22.86 & 22.25 & \\
\hline cep082 & $0^{\mathrm{h}} 55^{\mathrm{m}} 01^{\mathrm{s}} .56$ & $-37^{\circ} 35^{\prime} 43^{\prime \prime} 6$ & 11.5 & 1516.9 & 23.26 & 22.57 & \\
\hline cep083 & $0^{\mathrm{h}} 54^{\mathrm{m}} 59.60$ & $-37^{\circ} 47^{\prime} 31^{\prime \prime} 7$ & 11.4 & 1425.1 & 22.47 & 21.92 & blend \\
\hline cep084 & $0^{\mathrm{h}} 55^{\mathrm{m}} 10^{\mathrm{s}} 22$ & $-37^{\circ} 44^{\prime} 05^{\prime \prime} 8$ & 11.3 & 1496.7 & 22.77 & 21.97 & \\
\hline cep085 & $0^{\mathrm{h}} 54^{\mathrm{m}} 19.73$ & $-37^{\circ} 37^{\prime} 47^{\prime \prime} .7$ & 11.2 & 1496.0 & 22.60 & 22.21 & \\
\hline сер086 & $0^{\mathrm{h}} 55^{\mathrm{m}} 12^{\mathrm{s}} 49$ & $-37^{\circ} 36^{\prime} 11^{\prime \prime} 3$ & 10.6 & 1409.3 & 23.35 & 22.61 & \\
\hline cep087 & $0^{\mathrm{h}} 54^{\mathrm{m}} 55^{\mathrm{s}} .63$ & $-37^{\circ} 32^{\prime} 20^{\prime \prime} 2$ & 9.5 & 1496.6 & 22.96 & 22.45 & \\
\hline cep088 & $0^{\mathrm{h}} 55^{\mathrm{m}} 29.72$ & $-37^{\circ} 46^{\prime} 18^{\prime \prime} 6$ & 9.38 & 1484.53 & 23.26 & 22.51 & \\
\hline сер089 & $0^{\mathrm{h}} 54^{\mathrm{m}} 23.59$ & $-37^{\circ} 35^{\prime} 46^{\prime \prime} 4$ & 9.37 & 1521.73 & 22.82 & 22.16 & \\
\hline сер090 & $0^{\mathrm{h}} 55^{\mathrm{m}} 22^{\mathrm{s}} 61$ & $-37^{\circ} 34^{\prime} 23^{\prime \prime} 7$ & 9.3 & 1491.8 & 22.71 & 22.33 & \\
\hline сер091 & $0^{\mathrm{h}} 54^{\mathrm{m}} 24^{\mathrm{s}} 31$ & $-37^{\circ} 35^{\prime} 46^{\prime \prime} 0$ & 9.2 & 1481.8 & 23.26 & 22.62 & \\
\hline сер092 & $0^{\mathrm{h}} 55^{\mathrm{m}} 14^{\mathrm{s}} 60$ & $-37^{\circ} 36^{\prime} 02^{\prime \prime} 7$ & 9.07 & 1491.47 & 23.07 & 22.55 & \\
\hline cep093 & $0^{\mathrm{h}} 55^{\mathrm{m}} 01.83$ & $-37^{\circ} 37^{\prime} 52^{\prime \prime} 1$ & 9.06 & 1405.52 & 23.02 & 22.58 & \\
\hline cep094 & $0^{\mathrm{h}} 54^{\mathrm{m}} 50.87$ & $-37^{\circ} 35^{\prime} 49^{\prime \prime} 1$ & 8.89 & 1400.93 & 22.92 & 22.23 & blend \\
\hline cep095 & $0^{\mathrm{h}} 55^{\mathrm{m}} 01^{\mathrm{s}} .43$ & $-37^{\circ} 37^{\prime} 42^{\prime \prime} .5$ & 8.85 & 1547.45 & 22.93 & 22.11 & \\
\hline cep096 & $0^{\mathrm{h}} 55^{\mathrm{m}} 54^{\mathrm{s}} .92$ & $-37^{\circ} 46^{\prime} 39^{\prime \prime} 2$ & 8.76 & 1543.29 & 23.01 & 22.36 & \\
\hline cep097 & $0^{\mathrm{h}} 54^{\mathrm{m}} 25.36$ & $-37^{\circ} 31^{\prime} 58^{\prime \prime} .7$ & 8.46 & 1424.44 & 22.89 & 22.36 & \\
\hline сер098 & $0^{\mathrm{h}} 55^{\mathrm{m}} 40.50$ & $-37^{\circ} 37^{\prime} 47^{\prime \prime} 6$ & 8.42 & 1491.66 & 23.07 & 22.50 & \\
\hline cep099 & $0^{\mathrm{h}} 55^{\mathrm{m}} 34^{\mathrm{s}} .52$ & $-37^{\circ} 42^{\prime} 42^{\prime \prime} 4$ & 8.30 & 1436.72 & 23.04 & 22.48 & \\
\hline cep100 & $0^{\mathrm{h}} 55^{\mathrm{m}} 17^{\mathrm{s}} .53$ & $-37^{\circ} 37^{\prime} 24^{\prime \prime} .2$ & 8.07 & 1393.79 & 22.71 & 22.21 & \\
\hline сер101 & $0^{\mathrm{h}} 54^{\mathrm{m}} 40^{\mathrm{s}} 35$ & $-37^{\circ} 33^{\prime} 57^{\prime \prime} 8$ & 8.0 & 1481.5 & 22.53 & 21.95 & \\
\hline сер102 & $0^{\mathrm{h}} 54^{\mathrm{m}} 29.74$ & $-37^{\circ} 39^{\prime} 04^{\prime \prime} 0$ & 7.9 & 1526.3 & 23.28 & 22.80 & \\
\hline
\end{tabular}




\section{Previously Known Cepheids}

As mentioned before, a previous survey for variable stars in NGC 300 has already been conducted by Graham (1984). His observations, based on photographic plates taken at the prime focus of the CTIO $4 \mathrm{~m}$ telescope between November 1974 and October 1981, yielded a list of 34 variables or variable star candidates which included 18 Cepheids with derived periods, and another 5 objects suspected to be Cepheids, but without enough data for a period determination. Later Freedman et al. (1992) obtained CCD BVRI photometry at the same telescope for 16 of the Cepheids discovered by Graham . The baseline of their new observations was about 3 years, and as expected the quality of the CCD data was much higher than the previous photographic observations. However, in many cases the phase coverage of the resulting light curves was poor, making the determination of the mean brightnesses rather uncertain. Combining the photographic and their own CCD data, Freedman et al. (1992) had already obtained refined periods for some of the Cepheids.

From our new WFI data we rediscovered all 18 Cepheids reported by Graham (1984) (see Table 2). We also confirm 3 of his Cepheid candidates to be truly Cepheids (V7, V20, and V31). All the Graham variables were among the brightest objects in our database and have the most accurate photometry and the best coverage of their light curves. We also found that the object designated by Graham as V4 and classified by him as an eclipsing binary is another Cepheid with $\mathrm{P}=15.37 \mathrm{~d}$. Most probably the spurious classification was due to poor resolution of the photographic data. We did not confirm the variability of the objects marked as V14 and V19. Regarding the periods, our values generally agree, within the quoted uncertainties, with those obtained in the past. However, in the case of 5 variables (V4, V18, V21, V24, V25) we found a significantly different value for the period. The discrepancy in the period determination for V24 may be the result of a genuine period change for this variable during the last 26 years. Unfortunately the previous observations are too scarce and uncertain to confirm this beyond doubt. For V21 we obtained a period of about 24.0 days instead of 9.667 days derived previously by Freedman et al. However, the Freedman et al. observations for V21 do fit much better with our period than with that derived by themselves. The same happens in the case of star V25. For both of these Cepheids the previous data look much better when phased with our periods than with those

obtained before, and we were able to refine the periods (see below) combining our new with the previous data. The photographic data obtained for stars V4 and V18 are not accurate enough to be useful to improve the periods from the current new data.

Including our new observations, the time baseline since the first epoch observations is now 26 years, corresponding to about 76-1000 pulsation cycles for the Cepheids, which makes further improvement of the periods of these stars possible. Before doing this we need 
Table 2-Continued

\begin{tabular}{|c|c|c|c|c|c|c|c|}
\hline ID & RA (J2000) & DEC (J2000) & $\begin{array}{c}\mathrm{P} \\
{[\text { days }]}\end{array}$ & $\begin{array}{c}\mathrm{T}_{0}-2450000 \\
\text { [HJD] }\end{array}$ & $\begin{array}{r}<B> \\
{[\mathrm{mag}]}\end{array}$ & $\begin{array}{r}<V> \\
{[\mathrm{mag}]}\end{array}$ & Remarks \\
\hline cep103 & $0^{\mathrm{h}} 54^{\mathrm{m}} 35^{\mathrm{s}} .68$ & $-37^{\circ} 44^{\prime} 54^{\prime \prime} .2$ & 7.85 & 1524.82 & 23.32 & 22.78 & \\
\hline cep104 & $0^{\mathrm{h}} 54^{\mathrm{m}} 39.54$ & $-37^{\circ} 35^{\prime} 28^{\prime \prime} .0$ & 7.75 & 1409.22 & 23.34 & 22.77 & \\
\hline cep105 & $0^{\mathrm{h}} 54^{\mathrm{m}} 30.73$ & $-37^{\circ} 41^{\prime} 50^{\prime \prime} 6$ & 7.75 & 1524.68 & 23.11 & 22.61 & \\
\hline cep106 & $0^{\mathrm{h}} 54^{\mathrm{m}} 18.66$ & $-37^{\circ} 45^{\prime} 59^{\prime \prime} .2$ & 7.67 & 1400.70 & 23.06 & 22.49 & \\
\hline cep107 & $0^{\mathrm{h}} 54^{\mathrm{m}} 41^{\mathrm{s}} \cdot 41$ & $-37^{\circ} 44^{\prime} 02^{\prime \prime} 8$ & 7.65 & 1517.17 & 23.17 & 22.58 & \\
\hline cep108 & $0^{\mathrm{h}} 55^{\mathrm{m}} 28.33$ & $-37^{\circ} 34^{\prime} 40^{\prime \prime} .7$ & 7.61 & 1526.54 & 23.08 & 22.68 & \\
\hline cep109 & $0^{\mathrm{h}} 54^{\mathrm{m}} 06.59$ & $-37^{\circ} 35^{\prime} 43^{\prime \prime} .3$ & 7.38 & 1516.83 & 23.11 & 22.56 & \\
\hline cep110 & $0^{\mathrm{h}} 54^{\mathrm{m}} 39.57$ & $-37^{\circ} 41^{\prime} 17^{\prime \prime} 6$ & 7.15 & 1521.79 & 23.01 & 22.56 & blend \\
\hline cep111 & $0^{\mathrm{h}} 54^{\mathrm{m}} 07^{\mathrm{s}} \cdot 72$ & $-37^{\circ} 39^{\prime} 51^{\prime \prime} .9$ & 7.10 & 1521.52 & 22.99 & 22.59 & \\
\hline cep112 & $0^{\mathrm{h}} 55^{\mathrm{m}} 01.03$ & $-37^{\circ} 45^{\prime} 25^{\prime \prime} .2$ & 6.92 & 1545.83 & 23.43 & 22.93 & \\
\hline cep113 & $0^{\mathrm{h}} 55^{\mathrm{m}} 05.93$ & $-37^{\circ} 37^{\prime} 13^{\prime \prime} 1$ & 6.6 & 1526.8 & 22.97 & 22.40 & \\
\hline cep114 & $0^{\mathrm{h}} 55^{\mathrm{m}} 33^{\mathrm{s}} .15$ & $-37^{\circ} 37^{\prime} 01^{\prime \prime} 8$ & 6.49 & 1424.79 & 23.13 & 22.73 & \\
\hline cep115 & $0^{\mathrm{h}} 55^{\mathrm{m}} 01^{\mathrm{s}} .70$ & $-37^{\circ} 39^{\prime} 02^{\prime \prime} 1$ & 6.25 & 1521.65 & 23.08 & 22.57 & \\
\hline cep116 & $0^{\mathrm{h}} 54^{\mathrm{m}} 53.41$ & $-37^{\circ} 35^{\prime} 59^{\prime \prime} 1$ & 5.98 & 1521.71 & 23.79 & 23.08 & \\
\hline cep117 & $0^{\mathrm{h}} 54^{\mathrm{m}} 53.53$ & $-37^{\circ} 42^{\prime} 25^{\prime \prime} .6$ & 5.42 & 1433.79 & 23.02 & 22.48 & \\
\hline
\end{tabular}


Table 3. Cepheid Candidates in NGC 300

\begin{tabular}{|c|c|c|c|c|c|c|c|}
\hline ID & RA (J2000) & DEC (J2000) & $\begin{array}{c}\mathrm{P} \\
{[\text { days] }}\end{array}$ & $\begin{array}{c}\mathrm{T}_{0}-2450000 \\
{[\mathrm{HJD}]}\end{array}$ & $\begin{array}{r}<B> \\
{[\mathrm{mag}]}\end{array}$ & $\begin{array}{l}<V> \\
{[\mathrm{mag}]}\end{array}$ & Remarks \\
\hline сер118 & $0^{\mathrm{h}} 54^{\mathrm{m}} 40^{\mathrm{s}} 06$ & $-37^{\circ} 37^{\prime} 53^{\prime \prime} 1$ & 13.8 & 1525.1 & 22.76 & 22.11 & \\
\hline cep119 & $0^{\mathrm{h}} 54^{\mathrm{m}} 16^{\mathrm{s}} \cdot 94$ & $-37^{\circ} 37^{\prime} 23^{\prime \prime} .5$ & 13.4 & 1482.8 & 22.81 & 22.12 & \\
\hline cep120 & $0^{\mathrm{h}} 54^{\mathrm{m}} 20^{\mathrm{s}} 33$ & $-37^{\circ} 34^{\prime} 24^{\prime \prime} 1$ & 11.82 & 1430.98 & 22.76 & 22.64 & blend \\
\hline cep121 & $0^{\mathrm{h}} 54^{\mathrm{m}} 32^{\mathrm{s}} .70$ & $-37^{\circ} 37^{\prime} 47^{\prime \prime} .8$ & 11.3 & 1526.6 & 23.14 & 22.37 & \\
\hline cep122 & $0^{\mathrm{h}} 54^{\mathrm{m}} 59^{\mathrm{s}} .91$ & $-37^{\circ} 36^{\prime} 27^{\prime \prime} 9$ & 10.5 & 1517.1 & 22.77 & 22.39 & blend \\
\hline cep123 & $0^{\mathrm{h}} 55^{\mathrm{m}} 34^{\mathrm{s}} .95$ & $-37^{\circ} 41^{\prime} 22^{\prime \prime} 4$ & 8.7 & 1492.0 & 22.75 & 22.20 & blend \\
\hline cep124 & $0^{\mathrm{h}} 54^{\mathrm{m}} 50.09$ & $-37^{\circ} 42^{\prime} 18^{\prime \prime} 3$ & 8.3 & 1433.8 & 22.95 & 22.38 & blend \\
\hline сер125 & $0^{\mathrm{h}} 54^{\mathrm{m}} 26^{\mathrm{s}} 88$ & $-37^{\circ} 38^{\prime} 39^{\prime \prime} 7$ & 7.97 & 1524.61 & 23.05 & 22.72 & \\
\hline cep126 & $0^{\mathrm{h}} 55^{\mathrm{m}} 22^{\mathrm{s}} .91$ & $-37^{\circ} 43^{\prime} 48^{\prime \prime} 3$ & 7.40 & 1488.55 & 23.14 & 22.72 & \\
\hline cep127 & $0^{\mathrm{h}} 54^{\mathrm{m}} 20^{\mathrm{s}} .58$ & $-37^{\circ} 34^{\prime} 45^{\prime \prime} 3$ & 7.30 & 1431.85 & 23.12 & 22.82 & \\
\hline cep128 & $0^{\mathrm{h}} 55^{\mathrm{m}} 33^{\mathrm{s}} 81$ & $-37^{\circ} 42^{\prime} 39^{\prime \prime} 4$ & 6.15 & 1545.47 & 23.13 & 22.81 & \\
\hline сер129 & $0^{\mathrm{h}} 54^{\mathrm{m}} 49^{\mathrm{s}} 47$ & $-37^{\circ} 43^{\prime} 58^{\prime \prime} 8$ & 5.37 & 1516.64 & 22.83 & 22.47 & \\
\hline
\end{tabular}


Table 4. Individual B and V Observations

\begin{tabular}{|c|c|c|c|c|}
\hline object & filter & HJD-2450000 & mag & $\sigma_{m a g}$ \\
\hline cep001 & B & 1390.790 & 20.786 & 0.027 \\
\hline сер001 & B & 1391.939 & 20.826 & 0.011 \\
\hline сер001 & B & 1392.777 & 20.839 & 0.027 \\
\hline сер001 & B & 1393.831 & 20.812 & 0.029 \\
\hline сер001 & B & 1400.918 & 20.882 & 0.011 \\
\hline сер001 & B & 1404.815 & 20.979 & 0.013 \\
\hline сер001 & B & 1408.822 & 21.039 & 0.010 \\
\hline сер001 & B & 1424.819 & 21.455 & 0.038 \\
\hline сер001 & B & 1431.808 & 21.636 & 0.011 \\
\hline сер001 & B & 1433.803 & 21.584 & 0.021 \\
\hline сер001 & $\mathrm{B}$ & 1436.809 & 21.705 & 0.015 \\
\hline сер001 & $\mathrm{B}$ & 1438.815 & 21.768 & 0.020 \\
\hline сер001 & $\mathrm{B}$ & 1481.586 & 20.342 & 0.017 \\
\hline сер001 & B & 1484.586 & 20.105 & 0.013 \\
\hline сер001 & B & 1488.546 & 20.162 & 0.013 \\
\hline сер001 & B & 1491.565 & 20.241 & 0.011 \\
\hline сер001 & B & 1496.517 & 20.414 & 0.029 \\
\hline сер001 & B & 1498.566 & 20.511 & 0.014 \\
\hline сер001 & B & 1516.594 & 20.938 & 0.010 \\
\hline сер001 & B & 1519.570 & 21.004 & 0.006 \\
\hline cep001 & B & 1521.572 & 21.051 & 0.009 \\
\hline сер001 & B & 1524.577 & 21.081 & 0.010 \\
\hline сер001 & B & 1526.549 & 21.197 & 0.022 \\
\hline сер001 & B & 1528.613 & 21.104 & 0.038 \\
\hline сер001 & B & 1531.572 & 21.086 & 0.049 \\
\hline сер001 & B & 1543.549 & 21.514 & 0.027 \\
\hline сер001 & B & 1545.636 & 21.469 & 0.027 \\
\hline сер001 & $\mathrm{B}$ & 1547.560 & 21.596 & 0.027 \\
\hline
\end{tabular}

Note. - The complete version of this table is in the electronic edition of the Journal. The printed edition contains only the B data for the Cepheid variable cep001. 
to make sure that all the data sets share the same photometric zero point. Walker (1988) had already shown and corrected a zero point and scale error in Graham's photometric data. Using this correction, we tied the Graham data to Freedman's CCD system using the relations derived by Freedman et al. (1992). Finally, we need to check if the Freedman et al. (1992) photometry is not significantly different from ours. We did this by comparing the intensity mean magnitudes derived from our measurements to those given by Freedman et al., for the common stars. Fig. 6 presents this comparison for 8 Cepheids with reasonably good photometry from Freedman et al. It is seen that both data sets yield mean magnitudes which are in very good agreement. We also checked the agreement between individual observations from these two data sets. An example is given in Fig. 7 for the variable V3. It is seen that a systematic zero point offset, if any, is very small, and that the individual observations generally agree very well. It is also worth noting that apart from their much larger scatter, the photographic data fit also quite well our observations.

After making sure that there are no significant shifts in photometric zero points of the three available data sets, we phased all the data for common objects with the best available period (usually that from Freedman et al., (1992)), looking for phase shifts to our present new data. In 15 cases we found clear shifts in the phase indicating the need to adjust the period. The periods of these stars given in Table 1 are already the improved periods resulting from a combination of the available data sets. For 7 remaining Cepheids, the long gaps between the individual data sets, the accuracy of the available periods, the large scatter in the photographic data, and a very limited number of previous CCD observations (if available) did not allow us to refine the periods that we derived from our data alone.

\section{Summary and Discussion}

Results are presented of a wide-field multi-color photometric monitoring of NGC 300. Based on observations obtained on 29 nights during a 5.3 month period, 117 Cepheids and 12 Cepheid candidates were discovered from images in B and V bands. Their periods range between 115 and 5.42 days. For each object we provide equatorial coordinates, period, intensity mean $\mathrm{B}$ and $\mathrm{V}$ magnitudes and phased light curves. The accuracy of the derived periods is generally about $0.5-1 * 10^{-2} * \mathrm{P}$. We have re-discovered all previously known Cepheid variables, and for many of these the periods could be improved by combining the available data sets. The photometric zero points in both $\mathrm{B}$ and $\mathrm{V}$ are secure to within 0.03 mag.

The spatial distribution of the detected Cepheids is shown in Fig. 1. It can be appreciated that we were able to detect Cepheids even in the very dense regions near the 
center of NGC 300. Since the Cepheid distribution traces the spiral arms of the galaxy, most of our Cepheids lie in dense fields and are most probably blended, to some degree, with unresolved stars. This blending problem will be discussed in more detail in a forthcoming paper in which we will derive the distance of NGC 300 based on Cepheid PL relations.

In Fig. 8, we show the location of the detected Cepheids on the observed V, B-V color-magnitude diagram constructed from our data. The Cepheids delineate the typical Cepheid instability strip. Fig. 7 also indicates that the completeness of our Cepheid search drops suddenly for objects fainter than about 22.5 mag in V. For magnitudes brighter than this, we expect the survey to be near-complete. The corresponding period cutoff is close to 10 days, in the sense that our survey should have discovered a very large fraction of the Cepheids in NGC 300 with periods larger than 10 days.

Our Cepheid catalog contains 22 entries common to the list of variable stars prepared by Graham (1984). Sixteen of the Cepheids had also accurate (albeit sometimes sparse) previous CCD photometry (Freedman et al. 1992). We found very good agreement between the zero points in $\mathrm{B}$ and $\mathrm{V}$ of our data and those presented by Freedman et al. Using combined data sets, the periods of common objects were examined and improved whenever possible.

Fig. 9 presents the period distribution for all the 117 Cepheids in our catalog. There are three evident peaks, corresponding to periods of about 8, 14 and 24 days. Their existence and locations do not depend on the size of the binning we are using. A precise interpretation of the physical meaning of these peaks is difficult (and beyond the scope of this paper) but one possibility is that they reflect the fact that the Cepheids we are observing in NGC 300 have been formed during different bursts of star formation, in different places in the galaxy.

Finally, we show the period-luminosity relations in B and V derived from our data in Fig. 10. For periods below about 10 days, the data are clearly affected by a Malmquist bias-only the brighter Cepheids, at any given period below 10 days, have been detected in our survey. A detailed analysis of the PL relations to determine the distance of NGC 300 will be the subject of a forthcoming paper, which will include the presentation and analysis of photometric data obtained at longer wavelengths.

We are very grateful to the European Southern Observatory for providing large amounts of observing time with the Wide Field Camera on the $2.2 \mathrm{~m} \mathrm{ESO/MPI}$ telescope to this survey. It is a great pleasure to thank the $2 \mathrm{p} 2$ team for expert support. Among the astronomers who have been especially helpful with the observations we would like to thank H. Boehnhardt, P. Leisy, J. Brewer, E. Pompei, D. Bersier, H. Jones, P. Francois 
and T. Augusteijn. Pierre Leisy and L. Rizzi were very helpful with the data reductions. Furthermore, we would like to thank Drs Andrzej Udalski, Michał Szymański, and Grzegorz Pojmański for making their computer programs available and valuable discussion. WG would like to acknowledge financial support from Fondecyt grant 8000002, and express his thanks to the Centrum fuer internationale Migration und Entwicklung (CIM) in Germany for providing funds for a Sun Ultra 60 workstation on which most of the present data analysis was carried out. We also acknowledge use of the Digitized Sky Survey which was produced at the Space Telescope Science Institute based on photographic data obtained with the UK Schmidt Telescope, operated by the Royal Observatory Edinburgh.

\section{REFERENCES}

Burstein, D., \& Heiles, C. 1984, ApJS, 54, 33

Bresolin, F., Gieren, W., Kudritzki, R.P., Pietrzyński, G., \& Przybilla, N. 2001, ApJ, submitted

Freedman, W.L., Madore, B.F., Hawley, S.L., Horowitz, I.K., Mould, J., Navarrete. M., \& Sallmen, S. 1992 ApJ, 396, 80

Freedman, W.L., et al. 2001, ApJ, 553, 47

Graham, J.A. 1984, AJ, 89, 1332

Kudritzki, R.P., Puls, J., Lennon, D.J., Venn, K.A., Reetz, J., Najarro, F., McCarthy, J.K., \& Herrero, A., 1999, A\&A, 350, 970

Mould, J.R., et al. 2000, ApJ, 528, 655

Pietrzyński, G., Gieren, W., \& Udalski, A., 2001, PASP, submitted

Udalski, A., Szymański, M., Kubiak, M., Pietrzyński, G., Woźniak, P., \& Żebruń, K. 1998, Acta Astron., 48, 147

Walker, R.A. 1988, PASP, 100, 949

Walker, R.A. 1995, PASP, 107, 683 
Fig. 1.- DSS map of NGC300. Red and yellow points corespond to the positions of detected Cepheids with periods smaller and larger than 15 days, respectively. North is up and east to the left. The field of view is about $34 \times 34$ arcmin. This figure is too big to present it here.
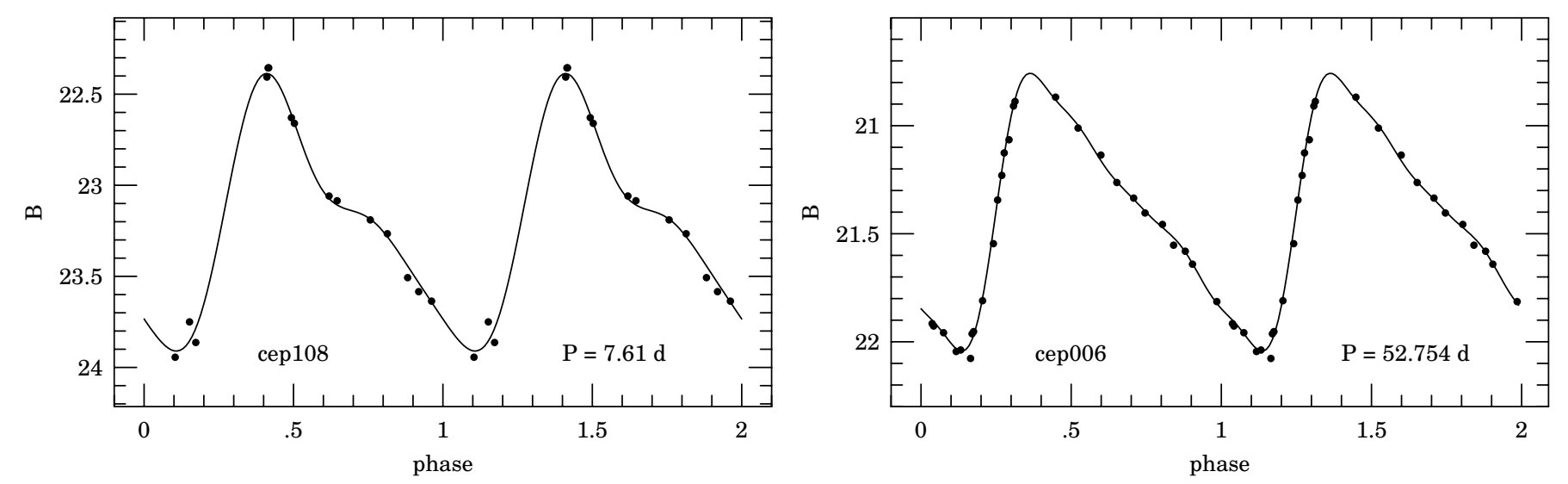

Fig. 2.- Two exemplary B light curves of Cepheid variables in NGC 300, with fitted Fourier series of order 3 (left panel), and 5 (right panel). Phase points are repeated for clarity. It is seen that good fits can be derived even for faint Cepheids with relatively noisy light curves. 

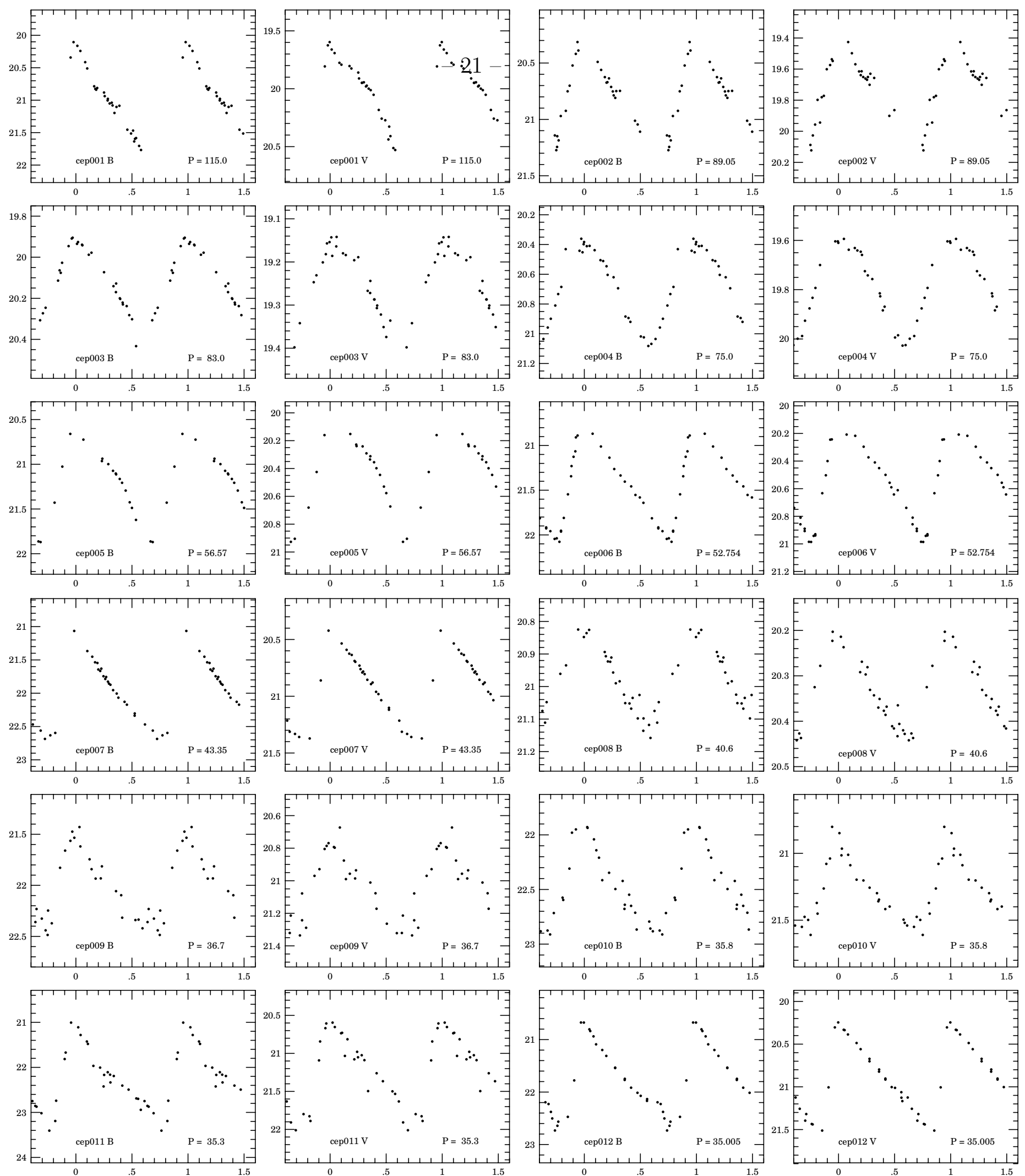

Fig. 3.- Sample of light curves in B and V bands for Cepheids in NGC 300 detected in our survey. Each point corresponds to a mean from 3-5 consecutive $360 \mathrm{~s}$ exposures from a given night. 
Fig. 4.- Light curves of additional Cepheid candidates in NGC 300. Not included here.

Fig. 5.- Finding charts for Cepheids and Cepheid candidates (not included here). The size of each map is about $0.5 \times 0.5$ arcmin. North is up and East to the left.

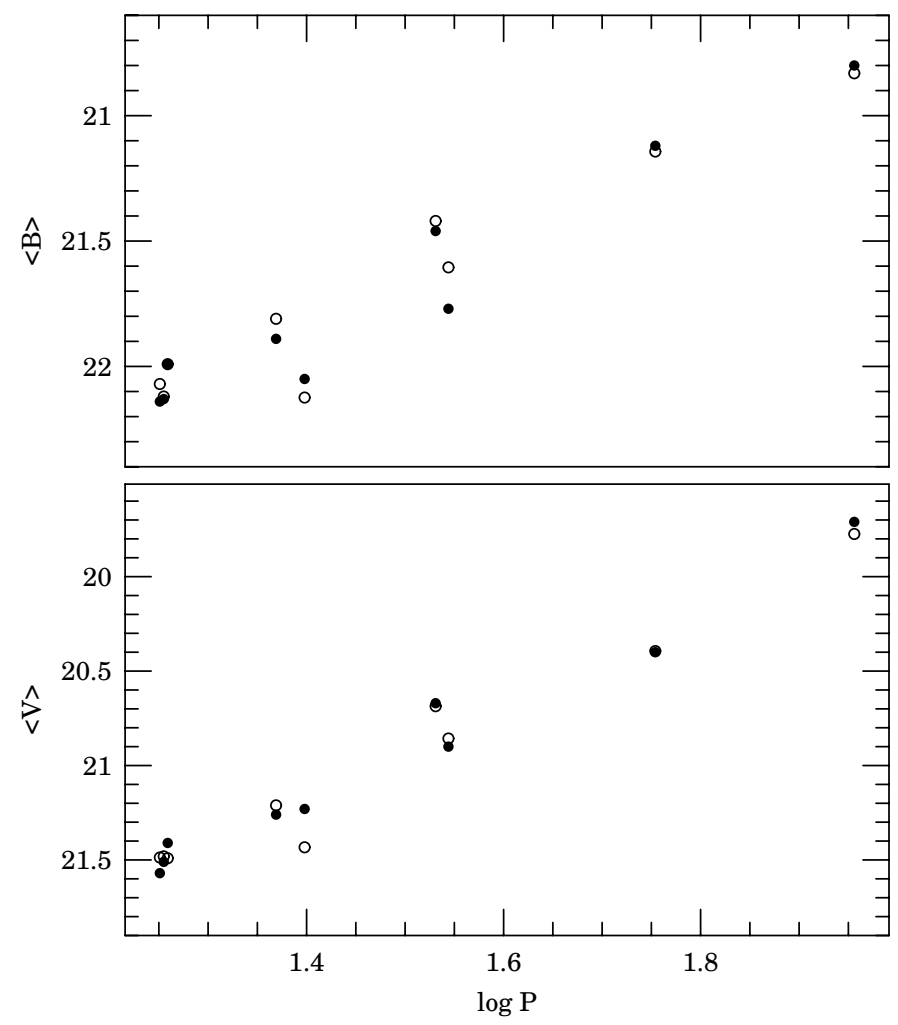

Fig. 6. - Mean B and V brightness versus logarithm of period for 8 Cepheids common to our catalog and Freedman et al. (1992). Filled and open circles correspond to our, and Freedman et al. results, respectively. 


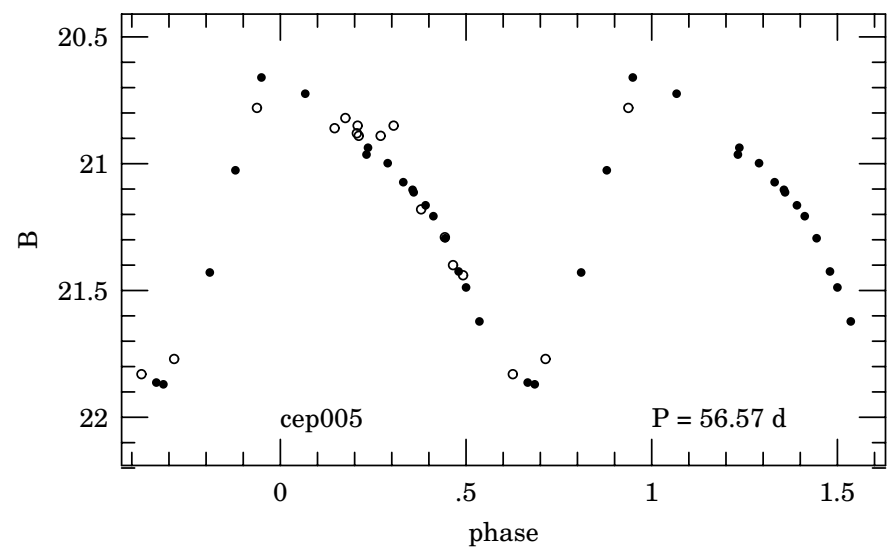

Fig. 7.- Combined B light curve for the Cepheid cep005. Filled and open circles correspond to our, and Freedman et al. data, respectively. The good agreement between the two data sets is demonstrated. 


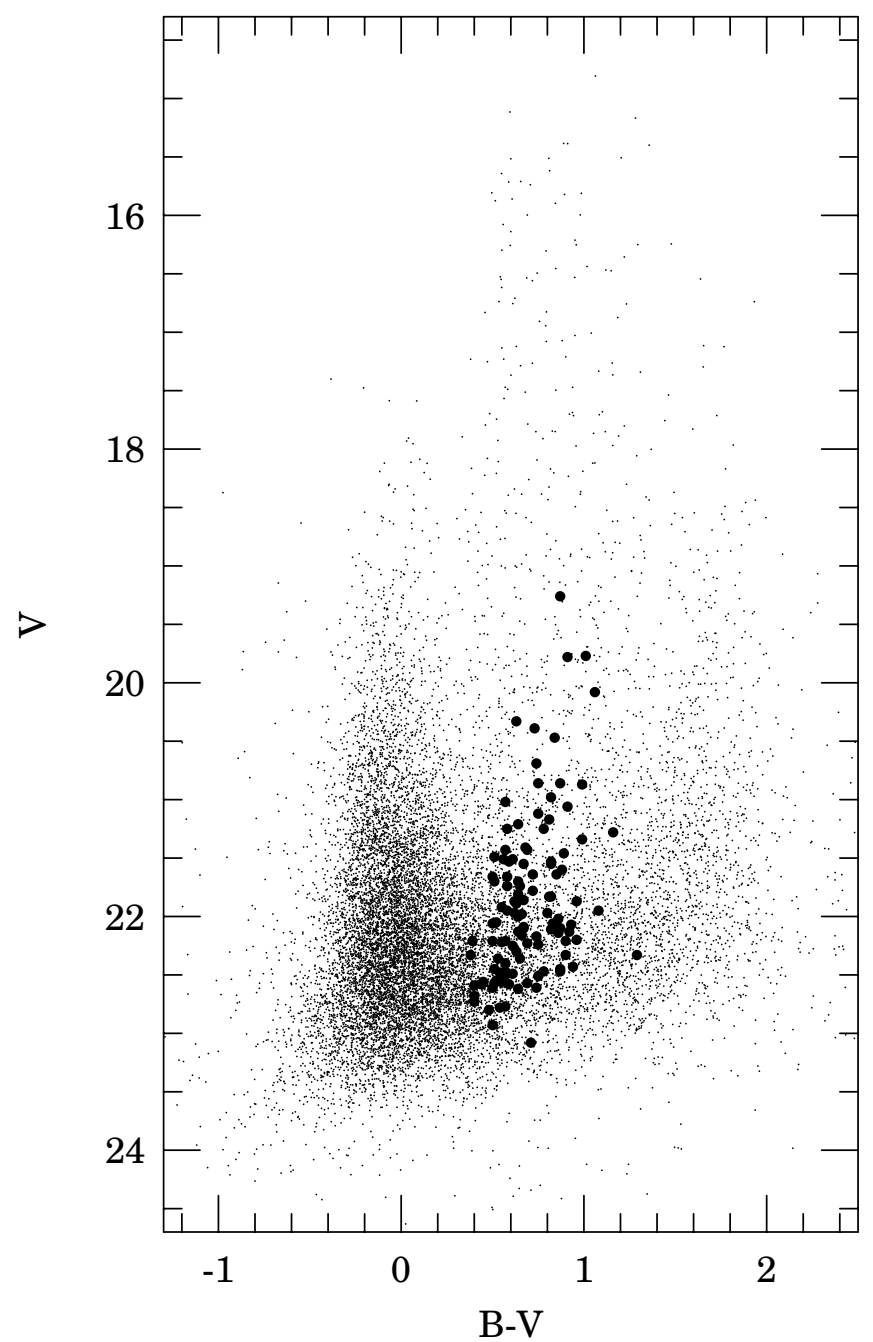

Fig. 8.- V, B-V color-magnitude diagram for stars in NGC 300 with photometry from our WFI data. The positions of the discovered Cepheids are marked with the filled circles. 


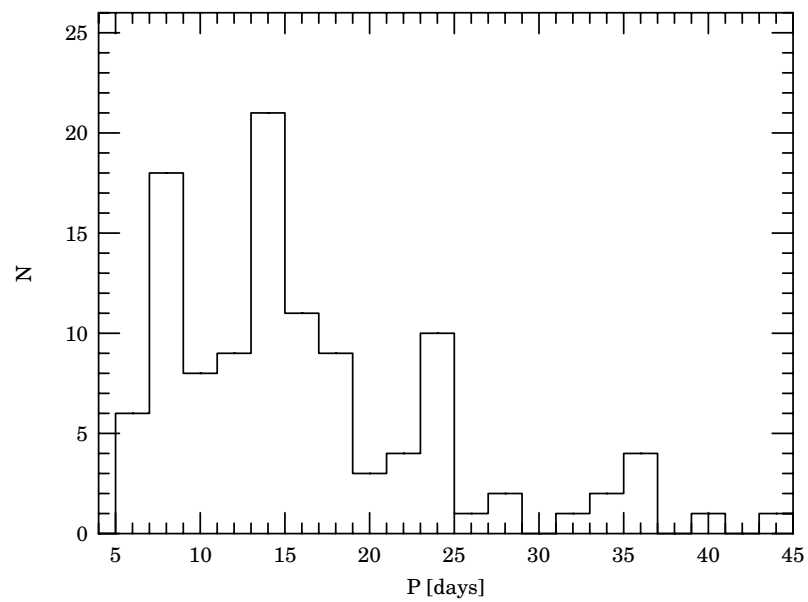

Fig. 9.- Period distribution of Cepheids in NGC 300 from our catalog. 

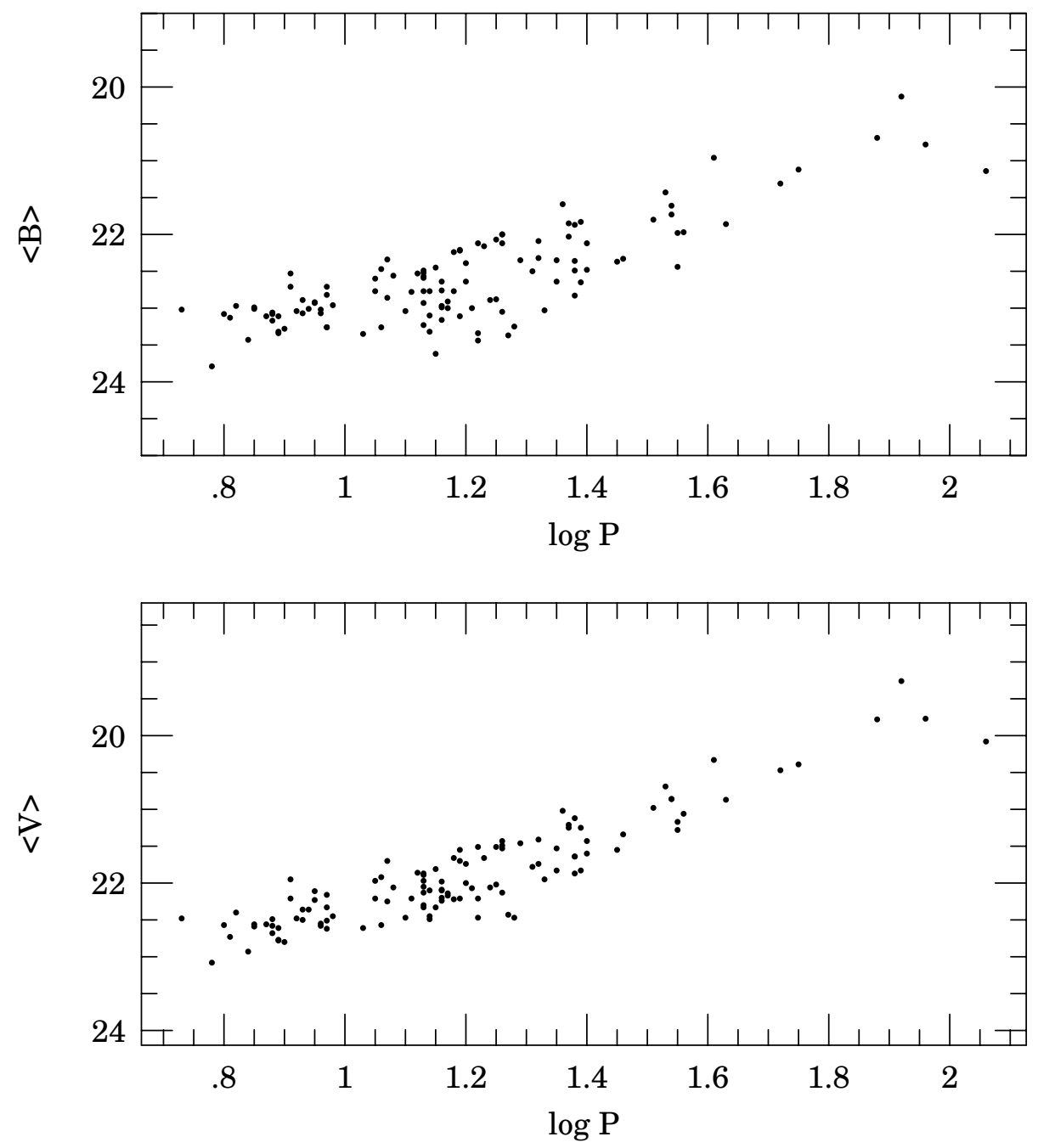

Fig. 10.- PL relations for B and V magnitudes from the Cepheids in NGC 300 detected in our survey. No absorption corrections have been applied to the mean magnitudes. 